\title{
Wake-induced bending of two-dimensional plasma crystals
}

\author{
T. B. Röcker, ${ }^{1, a)}$ A. V. Ivlev, ${ }^{1, b}$, S. K. Zhdanov, ${ }^{1}$ L. Couëdel, ${ }^{2}$ and G. E. Morfill ${ }^{1}$ \\ 1) Max Planck Institute for Extraterrestrial Physics, 85741 Garching, Germany \\ ${ }^{2)}$ CNRS, Aix-Marseille-Université, Laboratoire de Physique des Intéractions Ioniques et Moléculaires, \\ 13397 Marseille Cedex 20, France
}

(Dated: 16 January 2021)

It is shown that the wake-mediated interactions between microparticles in a two-dimensional plasma crystal affect the shape of the monolayer, making it non-flat. The equilibrium shape is calculated for various distributions of the particle number density in the monolayer. For typical experimental conditions, the levitation height of particles in the center of the crystal can be noticeably smaller than at the periphery. It is suggested that the effect of wake-induced bending can be utilized in experiments, to deduce important characteristics of the interparticle interaction.

PACS numbers: 52.27.Lw, 52.27.Gr

\section{INTRODUCTION}

Two-dimensional (2D) complex plasmas have been actively investigated in ground-based experiments since the two decades $\underline{1-5}$. The focus has been made on experiments with crystalline and liquid monolayers, representing an excellent natural model system for studies of generic phenomena occurring in classical 2D liquids and solids 6.7 . Such monolayers are usually obtained in radio-frequency (rf) plasma discharge chambers $\underline{\underline{8}-13}$, where the negatively charged monodisperse microparticles levitate above the horizontal rf electrode due to the balance between the electrostatic force (exerted in the electrode sheath via an inhomogeneous vertical electric field) and gravity.

The sheath field induces a strong vertical plasma flow toward the electrode, and each microparticle acts as the lens causing the flowing ions to focus downstream from it. This results in the formation of plasma wakes "attached" to microparticles $14-22$. The wakes exert attractive forces on the neighboring particles and make the pair interactions non-reciprocal. Nevertheless, the collective behavior of such monolayers remains exactly conservative (i.e., apart from a frictional neutral-gas damping, it can still be described by an effective Hamiltonian) until a certain threshold in the particle number density is reached. This threshold depends on the strength of the vertical confinement and identifies the onset of the modecoupling instability $(\mathrm{MCI})^{23-29}$. In the unstable regime the particles acquire anomalous kinetic energy which is converted from the flowing plasma (due to resonance coupling between the horizontal and vertical wave modes, mediated by non-reciprocal particle-wake interactions). If the MCI threshold is reached, the instability can only be suppressed by frictional damping, e.g., by increasing the gas pressure.

Theoretical studies of $2 \mathrm{D}$ complex plasmas usually assume that monolayers are flat, i.e., all particles levitate

\footnotetext{
a) Electronic mail: tbr@mpe.mpg.de

b) Electronic mail: ivlev@mpe.mpg.de
}

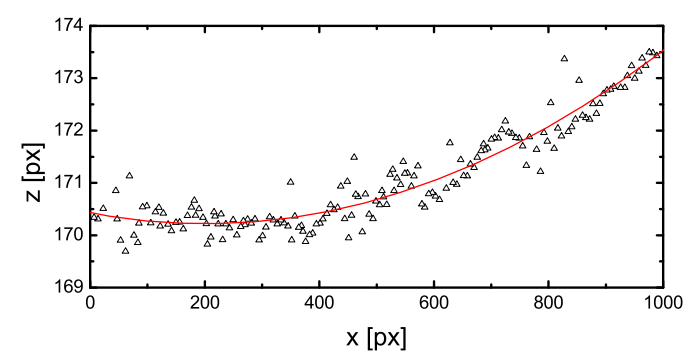

FIG. 1. Illustration of bending of a 2D plasma crystal seen in experiments. A horizontal disk-shaped 2D crystalline monolayer was formed by monodisperse melamine-formaldehyde particles of $9.19 \mu \mathrm{m}$ diameter, levitated in an argon discharge above a flat $\mathrm{rf}$ electrode of about $20 \mathrm{~cm}$ diameter. Particles were illuminated by a vertical thin $(\simeq 2$ interparticle distances) laser sheet passing through the center of the monolayer. Shown are the levitation heights of the traced individual particles in the illuminated region, the solid line is a parabolic fit. The levitation height $z$ turns out to be dependent on the radial distance $x$ from the center, where $z(x)$ attains a minimum (and where the areal density of particles is the highest). The pixel size is $57 \mu \mathrm{m}$, the vertical coordinate is measured from the electrode surface.

on the same height, and that the number density is constant. In fact, the density normally has a maximum near the center (monolayers are typically disk-shaped) and decreases towards the periphery. This inhomogeneity is caused by a weak (radially-dependent) horizontal confinement, primarily due to edge effects in the discharg $\mathrm{e}^{30}-33$. The levitation height also varies across the monolayer - it turns out to be the lowest at the center, so that the monolayer has an observable curvature ("bending"), as illustrated in Fig. 11 To the best of our knowledge, the cause of the bending has never been investigated, e.g., it may be associated with the edge effects too.

We would like to point out a mechanism of the bending which must always operate in experiments with $2 \mathrm{D}$ 
complex plasmas: Along with the "individual" forces acting on each levitated particle (gravity, electrostatic, ion drag, thermophoretic, etc.), there is also a "collective" contribution from the wakes of the neighboring particles. Unlike the direct electrostatic interaction between charged particles, the wake-mediated interactions break the symmetry $\stackrel{34}{4}$. The forces from the wakes of the neighboring particles are pointed downwards and, obviously, the resulting net force should be dependent on the local particle density and configuration in the monolayer. Thus, the wake-mediated interactions should always cause bending of the monolayer, and one could expect that the effect should be the strongest in the center, where the particle density is the highest.

In this paper we analyze the effect of the wakemediated interactions on bending of $2 \mathrm{D}$ plasma crystals. We show that, under typical experimental conditions the magnitude of the effect can be quite significant: The difference between the levitation height in the center and at the periphery can be as large as $\sim 10 \%$ of the mean interparticle distance. We suggest that the bending phenomenon can be utilized in experiments, to deduce important characteristics of the interparticle interaction.

\section{BENDING}

Let us consider a monolayer composed of $N$ identical charged particles whose equilibrium is determined by the balance between the wake-mediated pair interactions and the forces of external confinement. The equilibrium configuration can be described by the "bending surface" $z\left(x_{i}, y_{i}\right)$, where $\left\{x_{i}, y_{i}\right\}$ are the equilibrium horizontal coordinates of the $i$ th particle $(1 \leq i \leq N)$.

The horizontal confinement is usually poorly known, and therefore determining the horizontal particle distribution "from the first principles" is hardly possible. On the other hand, the horizontal positions can be directly obtained from the experimental top-view observations. Hence, one can use the measured coordinates $\left\{x_{i}, y_{i}\right\}$ as the input for calculating the vertical displacement $z\left(x_{i}, y_{i}\right)$.

Parameters of the vertical confinement can be accurately measured in experiments with individual particles ${ }^{35}$. Typically, the confinement is harmonic to a good accuracy. It is characterized by the vertical eigenfrequency $\Omega_{\mathrm{v}}$; nonlinear terms can also be deduced and included in the analysis, for the sake of simplicity we neglect them here. For the interparticle interactions we implement a simple Yukawa/point-wake model28,36, which is characterized by the negative particle charge $-Q$, effective screening length $\lambda$, positive wake charge $q$, and wake length $\delta$. The results can be straightforwardly generalized for an arbitrary model of the wake potential.

In this section we normalize all lengths by $\lambda$, while the interaction potential and the vertical eigenfrequency are in units of the Debye energy scale $Q^{2} / \lambda$ and the dustlattice frequency $\sqrt{Q^{2} / M \lambda^{3}}$, respectively (where $M$ is

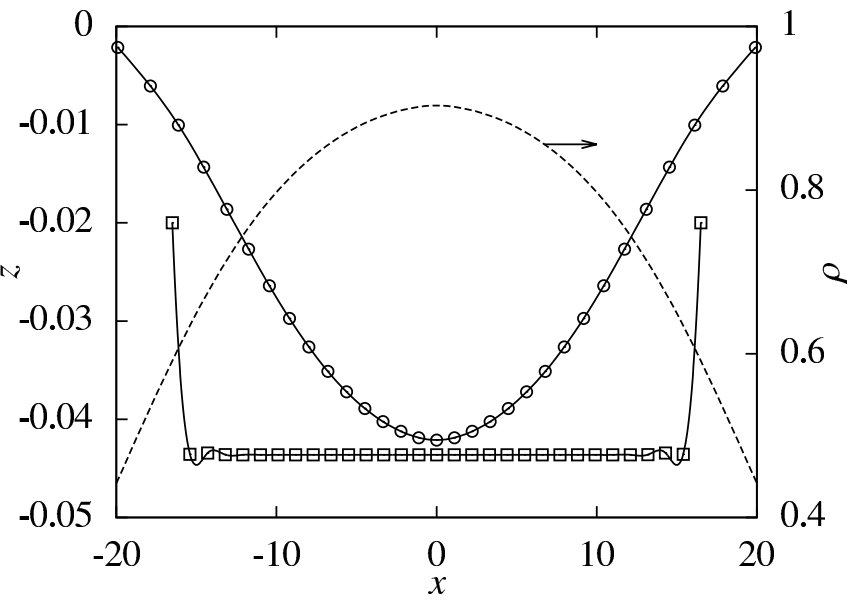

FIG. 2. Bending of a 1D particle string (dimensionless units). The vertical displacement $z\left(x_{i}\right)$ of 31 particles is plotted (relative to the equilibrium height of a single particle). Two examples are shown, corresponding to a constant (squares) and variable (circles) linear density $\rho$. The dashed line represents $\rho\left(x_{i}\right)$ for the latter case, the constant-density value coincides with its maximum. The results are for the screening parameter $\kappa=1.1$ (at the maximum) and the wake dipole moment $\tilde{q} \tilde{\delta}=0.24$, the eigenfrequency of vertical confinement $\Omega_{\mathrm{v}}=2.24$ corresponds to the MCI threshold (note that $z \propto \Omega_{\mathrm{v}}^{-2}$, see text for details).

the particle mass). The screening parameter $\kappa=\Delta / \lambda$ is defined for the mean interparticle distance $\Delta$ at the maximum density. Furthermore, we introduce the normalized wake charge $\tilde{q}=q / Q$ and length $\tilde{\delta}=\delta / \lambda$, so that the Yukawa/point-wake potential of a particle reads

$$
\phi(\mathbf{r})=\frac{e^{-r}}{r}-\tilde{q} \frac{e^{-r_{\delta}}}{r_{\delta}},
$$

where $r$ is the distance to the particle and $r_{\delta}=$ $\sqrt{x^{2}+y^{2}+(z+\tilde{\delta})^{2}}$ is the distance to the wake. The vertical displacement $z_{i} \equiv z\left(x_{i}, y_{i}\right)$ of the $i$ th particle is governed by the following equation readily derived from the vertical force balance:

$$
\left.\sum_{j \neq i} \frac{\partial \phi}{\partial z}\right|_{\mathbf{r}_{j}-\mathbf{r}_{i}}+\Omega_{\mathrm{v}}^{2} z_{i}=0,
$$

where the summation is over the neighbors. Thus, the bending surface is generally determined by the solution of $N$ coupled equations (2).

In order to demonstrate the generic properties of bending and, simultaneously, to highlight the important effect of neighbors, we shall start the consideration with a $1 \mathrm{D}$ particle string and then discuss 2D monolayers.

Figure 2 shows the "bending lines" $z\left(x_{i}\right)$ calculated from Eq. (21) for a 1D string comprised of $N=31$ particles. We present results for a constant density (squares) and variable density (circles). In the latter case the horizontal particle positions were deduced from molecular 
dynamics simulations (described in Refs $\underline{30,37}$ ). The resulting density distribution (dashed line) has a maximum at the center, where it is equal to the value used for the constant-density case.

Remarkably, for a constant-density case the string remains flat, i.e., practically all particles except those at the ends are shifted downwards as the whole. This indicates that the magnitude of the shift at a given particle density is mostly determined by the nearest neighbors, i.e., even for relatively small $(N \geq 5-6)$ clusters with equal spacing the vertical displacement is practically independent of $N$. In the case of variable density, the net force from the mutual interactions rapidly decreases toward the ends and, therefore, $\left|z_{i}\right|$ decreases as well, but the displacement at the center is practically the same as for the constant-density case.

Figure 3 illustrates bending of a 2D monolayer. The radial dependence of the vertical displacement is presented in Fig. [3a (solid line) for a given areal density distribution (dashed line), and the complementary results for a 1D string (dotted line, with the same linear density distribution) are also shown. Obviously, bending of monolayers and strings is qualitatively the same, but its magnitude $H$ (maximum vertical displacement) is about 3 times larger in the $2 \mathrm{D}$ case $(H \simeq 0.08)$. So, the magnitude of $H$ for a given density appears to be primarily determined by the lattice coordination number (whose ratio for $2 \mathrm{D}$ and $1 \mathrm{D}$ lattices is 3). Furthermore, Fig. 3b demonstrates that near the monolayers center, where the density is practically constant, the particles levitate at almost the same height.

A general dependence of the bending magnitude $H$ on the parameters of pair interaction and confinement can be derived from the following simple consideration: The bending is the result of interaction asymmetry due to the wake term in Eq. (11). For the latter, the dependence on $z$ is negligible when $\tilde{\delta}\left|d z / d r_{\mathrm{h}}\right| \ll \kappa$ (where $r_{\mathrm{h}}$ is the horizontal coordinate). As one can see from Fig. 3, $\left|d z / d r_{\mathrm{h}}\right|$ is typically very small $\left(\sim 10^{-2}\right)$, so this condition is always satisfied. Then the first term in Eq. (2) does not depend on $z_{i}$ and we get

$$
H=\frac{\tilde{q} \tilde{\delta}}{\Omega_{\mathrm{v}}^{2}} \sum_{i \neq 0} \frac{1+\xi_{i}}{\xi_{i}^{3}} e^{-\xi_{i}} \equiv \frac{\tilde{q} \tilde{\delta}}{\Omega_{\mathrm{v}}^{2}} \sigma(\kappa, \tilde{\delta})
$$

where $\xi_{i}=\sqrt{r_{\mathrm{h} i}^{2}+\tilde{\delta}^{2}}$ is the distance to the wake of the $i$ th neighbor. We immediately conclude that the bending magnitude is directly proportional to the effective wake charge and inversely proportional to the squared confinement frequency. One can further simplify the obtained expression by noting that the dependence of $\sigma$ on $\tilde{\delta}$ can be neglected when $\tilde{\delta}^{2} \ll \kappa^{2}$. Typically, the screening parameter $\kappa$ is about unity, so that for $\tilde{\delta} \lesssim 0.3$ the distance is reduced to $\xi_{i} \simeq r_{\mathrm{h} i}$. Hence, in practice $\sigma$ is a (strongly decreasing) function of $\kappa$ only, as shown in Fig. 4 (thin lines), and $H$ is proportional to the wake dipole moment $\tilde{q} \tilde{\delta}$. We note that the functions representing $2 \mathrm{D}$ and $1 \mathrm{D}$
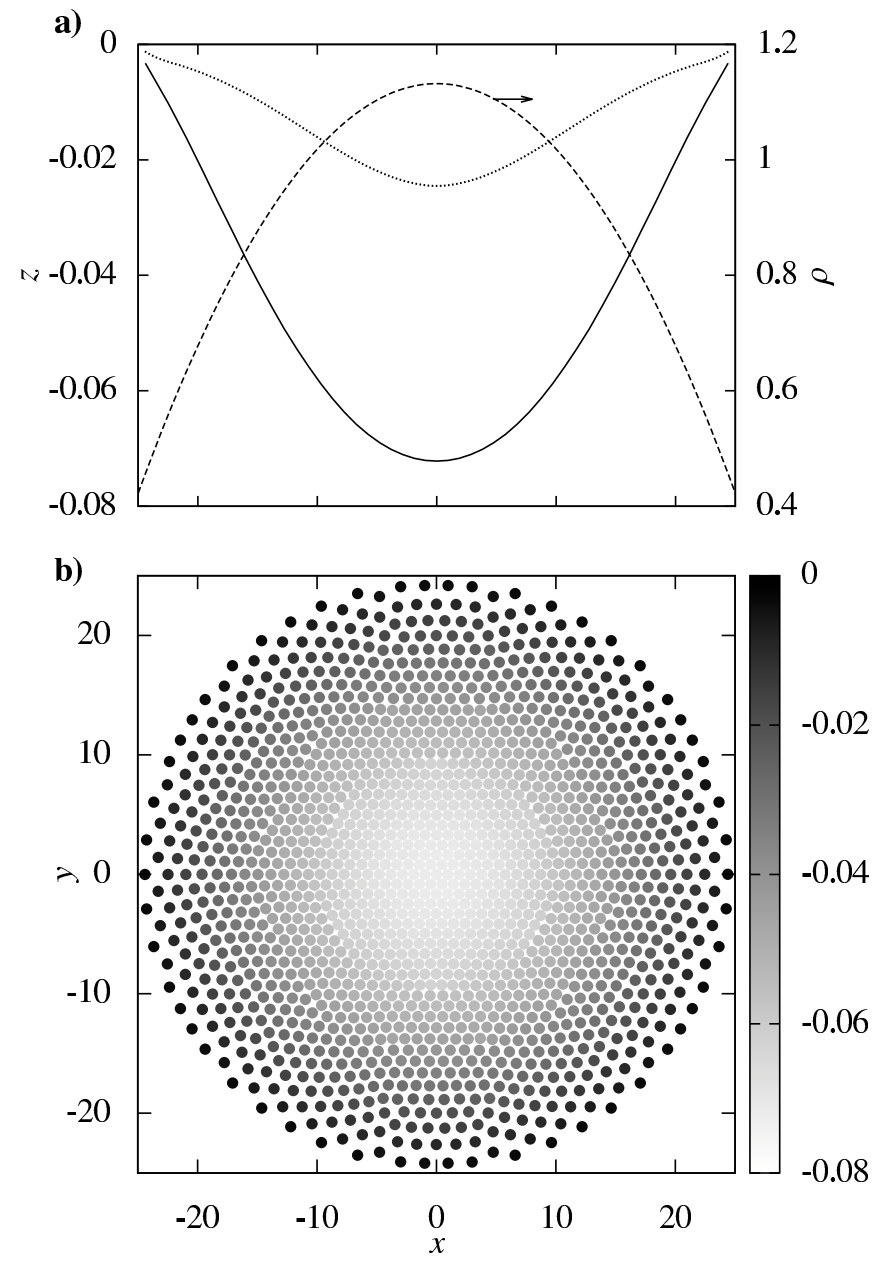

FIG. 3. Bending of a 2D crystalline monolayer (dimensionless units). The crystal has a form of an axially-symmetric disc composed of 1519 particles, the radial dependence of the (average) areal density $\rho$ is shown in (a) by the dashed line. The corresponding radial dependence of the vertical displacement $z$ is depicted by the solid line, and the bending line for a 1D particle string with the same linear density distribution is also shown for comparison (dotted line). The top view of the bent monolayer is presented in (b), where the magnitude of the local vertical displacement is grayscale-coded. The results are for the screening parameter $\kappa=0.91$ (at maximum density) and the wake dipole moment $\tilde{q} \tilde{\delta}=0.24$, the eigenfrequency of vertical confinement $\Omega_{\mathrm{v}}=4.22$ corresponds to the MCI threshold.

cases in Fig. 4 (solid and dashed lines, respectively) are similar and their ratio also approaches 3 for $\kappa \gtrsim 1$ (cf. bending profiles in Fig. (3a).

The strongest equilibrium bending is observed when $\Omega_{\mathrm{v}}$ is reduced down to the critical (threshold) confinement frequency $\Omega_{\text {cr }}$ corresponding to the onset of MCI. For $\tilde{\delta}^{2} \ll 1$, the combination $(1-\tilde{q})^{-1} \Omega_{\text {cr }}^{2}$ is a function of $\kappa$ only $\stackrel{28}{ }$, and this rather strong dependence turns out to be very similar to $\sigma(\kappa)$. Therefore, the maximum equilibrium magnitude of the bending is given by the following 


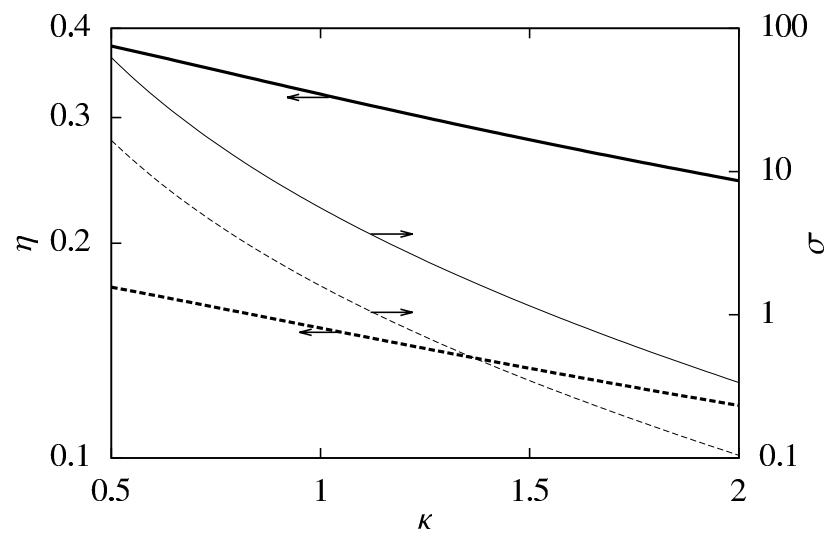

FIG. 4. Functions $\sigma(\kappa)$ (thin lines) and $\eta(\kappa)$ (thick lines) determining the universal dependencies for the bending magnitudes in Eqs. (3) and (4), respectively. The shown curves are for 2D monolayers (solid lines) and 1D strings (dashed lines), the results are obtained in the limit $\delta^{2} \ll 1$.

simple dependence:

$$
H_{\mathrm{cr}}=\tilde{q} \tilde{\delta} \frac{\sigma(\kappa)}{\Omega_{\mathrm{cr}}^{2}(\kappa)} \equiv \frac{\tilde{q} \tilde{\delta}}{1-\tilde{q}} \eta(\kappa)
$$

where $\eta(\kappa)$ is a relatively weak function shown in Fig. 4 (thick lines).

\section{CONCLUSION}

Bending of $2 \mathrm{D}$ plasma crystals is not only an interesting effect caused by the interaction non-reciprocity. We showed that its magnitude can be quite large, about one tenth of the effective screening length, which gives about $40-80 \mu \mathrm{m}$ for typical experiments with $2 \mathrm{D}$ complex plasmas 28 . This value is comparable with the thickness of the horizontal laser sheets used in experiments to illuminate levitated particles. Therefore, we suggest that the bending phenomenon can be utilized to deduce important characteristics of the interparticle interaction.

The only well-established experimental method to determine the screening length and the effective particle charge, $\sqrt{1-\tilde{q}} Q^{28}$, is based on fitting theoretical dispersion relations to experimental fluctuation spectra 27.38 . Another recently proposed ${ }^{36}$ method of mapping selfconsistent wake models to the effective dipole moment $\tilde{q} \tilde{\delta}$ allows us to relate the wake parameters to the screening parameter $\kappa$. However, the accuracy of this method has not been tested so far.

The results presented in this paper allow us to identify at least two simple algorithms to obtain certain combinations of the interaction parameters. For this, let us rewrite Eqs. (3) and (41) in the dimensional form:

- From Eq. (4) we derive

$$
\frac{q \delta}{Q-q}=H_{\mathrm{cr}} \eta^{-1}(\kappa)
$$

By using the value of $\kappa$ in the center of the monolayer (e.g., deduced from fitting of fluctuation spectra) as the input parameter and measuring the bending magnitude $H_{\text {cr }}$ at the onset of MCI, we obtain a combination of $Q, q$, and $\delta$. Since $\eta$ only weakly depends on $\kappa$, this algorithm provides fairly accurate results even if the accuracy of measuring $\kappa$ is poor.

- From Eq. (3) we get

$$
Q q \delta=\left(2 \pi f_{\mathrm{v}}\right)^{2} M H \lambda^{3} \sigma^{-1}(\kappa) .
$$

In this case, it is sufficient to measure $H$ at a given resonance frequency of a single particle, $f_{\mathrm{v}}$ (in units of $\mathrm{Hz}$ ), and also use $\lambda$ as the input parameter, which yields another combination of $Q, q$, and $\delta$. Since $\sigma(\kappa)$ is a strong function, this algorithm requires very accurate determination of $\lambda$. Note that one can divide the two obtained combinations to directly deduce the squared effective charge $(Q-q) Q$.

We conclude that the bending phenomenon commonly observed in experiments with 2D complex plasmas can provide us with a simple powerful method to deduce important characteristics of the interparticle interaction. On the other hand, of course, one should keep in mind that the bending could also be associated with other phenomena, e.g., with a possible inhomogeneity of the sheath electric field and/or particle charge in the horizontal direction. The magnitude (and even the sign) of such effects are unknown and can only be measured in dedicated experimental tests.

\section{ACKNOWLEDGMENTS}

We appreciate funding from the European Research Council under the European Unions Seventh Framework Programme/ERC Grant Agreement 267499, and from the French-German PHC PROCOPE Program/Project 28444XH/55926142. T.B.R. acknowledges Alexandra Heimisch for the helpful support.

${ }^{1}$ J.H. Chu and Lin I, Phys. Rev. Lett. 72, 4009 (1994).

${ }^{2}$ H. Thomas, G.E. Morfill, V. Demmel, J. Gorree, B. Feuerbacher, and D. Möhlmann, Phys. Rev. Lett. 73, 652 (1994).

${ }^{3}$ J. Maddox, Nature 370, 411 (1994).

${ }^{4}$ Y. Hayashi and K. Tachibana, Jpn. J. Appl. Phys. 33, L804 (1994).

${ }^{5}$ A. Melzer, T. Trottenberg, and A. Piel, Phys. Lett. A 191, 301 (1994).

${ }^{6}$ G.E. Morfill and A.V. Ivlev, Rev. Mod. Phys. 81, 1353 (2009).

${ }^{7}$ A. Ivlev, H. Löwen, G. Morfill, and C.P. Royall, Complex Plasmas and Colloidal Dispersions: Particle-Resolved Studies of Classical Liquids and Solids, World Scientific, Singapore (2012).

${ }^{8} \mathrm{U}$. Konopka, Wechselwirkungen geladener Staubteilchen in Hochfrequenzplasmen, Phd thesis (2000).

${ }^{9}$ H. Thomas and G. Morfill, Nature 379, 806 (1996).

${ }^{10}$ U. Konopka, L. Ratke, and H. M. Thomas, Phys. Rev. Lett. 79, 1269 (1997).

${ }^{11}$ D. Samsonov, J. Goree, Z.W. Ma, A. Bhattacharjee, H.M. Thomas, and G.E. Morfill, Phys. Rev. Lett. 83, 3649 (1999).

${ }^{12}$ U. Konopka, G.E. Morfill, and L. Ratke, Phys. Rev. Lett. 84, 891 (2000). 
${ }^{13}$ V. Nosenko, J. Goree, and A. Piel, Phys. Plasmas 13, 032106 (2006).

${ }^{14}$ O. Ishihara and S.V. Vladimirov, Phys. Plasmas 4, 69 (1997).

${ }^{15}$ M. Lampe, G. Joyce, G. Ganguli, and V. Gavrishchaka, Phys. Plasmas 7, 3851 (2000).

${ }^{16}$ A. Melzer, V.A. Schweigert, and A. Piel, Physica Scripta 61, 494 (2000).

${ }^{17}$ L.-J. Hou, Y.-N. Wang, and Z.L. Miskovic, Phys. Rev. E 64, 46406 (2001).

${ }^{18}$ S.V. Vladimirov, S.A. Maiorov, and O. Ishihara, Phys. Plasmas 10, 3867 (2003).

${ }^{19}$ A. Samarian, S. Vladimirov, and B. James, JETP Letters 82, 758 (2005).

${ }^{20}$ W.J. Miloch, Plasma Physics and Controlled Fusion 52, 124004 (2010).

${ }^{21}$ R. Kompaneets, U. Konopka, A.V. Ivlev, V. Tsytovich, and G. Morfill, Phys. Plasmas 14, 052108 (2007).

${ }^{22}$ A.V. Ivlev, S.K. Zhdanov and G.E. Morfill, Phys. Rev. E 71, 016405 (2005).

${ }^{23}$ A.V. Ivlev and G.E. Morfill, Phys. Rev. E 63, 016409 (2000).

${ }^{24}$ B. Liu, J. Goree, and Y. Feng, Phys. Rev. Lett. 105, 085004 (2010); B. Liu, J. Goree, and Y. Feng, Phys. Rev. Lett. 105, 269901 (2010).

${ }^{25}$ A.V. Ivlev, U. Konopka, and G.E. Morfill, Phys. Rev. E 68, 026405 (2003).
${ }^{26}$ S.K. Zhdanov, A.V. Ivlev, and G.E. Morfill, Phys. Plasmas 16, 083706 (2009).

${ }^{27}$ L. Couëdel, V. Nosenko, A.V. Ivlev, S.K. Zhdanov, H.M. Thomas, and G.E. Morfill, Phys. Rev. Lett. 104, 195001 (2010).

${ }^{28}$ L. Couëdel, S.K. Zhdanov, A.V. Ivlev, V. Nosenko, H.M. Thomas, and G.E. Morfill, Phys. Plasmas 18, 083707 (2011).

${ }^{29}$ T.B. Röcker, A.V. Ivlev, S.K. Zhdanov, and G.E. Morfill, Phys. Rev. E 89, 013104 (2014).

${ }^{30}$ S. Zhdanov, R.A. Quinn, D. Samsonov and G.E. Morfill, N. Journal Phys. 5, 74 (2003).

${ }^{31}$ R.A. Quinn, C. Cui, J. Goree and J.B. Pieper, Phys. Rev. E 53, R2049 (1996).

${ }^{32}$ T.E. Sheridan, Phys. Plasmas 16, 083705 (2009).

${ }^{33}$ C. Durniak, D. Samsonov, N.P. Oxtoby, J.P. Ralph and S. Zhdanov, IEEE Trans. Plasma Sci. 38, 2412 (2010).

${ }^{34}$ V. Steinberg, R. Sütterlin, A. V. Ivlev, and G. Morfill, Phys. Rev. Lett. 86, 4540 (2001).

${ }^{35}$ A. V. Ivlev, R. Sütterlin, V. Steinberg, M. Zuzic, and G. Morfill, Phys. Rev. Lett. 85, 4060 (2000).

${ }^{36}$ T.B. Röcker, S.K. Zhdanov, A.V. Ivlev, M. Lampe, G. Joyce, and G.E. Morfill, Phys. Plasmas 19, 073708 (2012).

${ }^{37}$ T.B. Röcker, Mode-coupling regimes in $2 D$ plasma crystals, $\mathrm{Phd}$ thesis (to be published).

${ }^{38} \mathrm{~S}$. Nunomura, J. Goree, S. Hu, X. Wang, A. Bhattacharjee, and K. Avinash, Phys. Rev. Lett. 89, 035001 (2002). 